\title{
THE FORMATION OF SINGLE AND BINARY NUCLEI OF PLANETARY NEBULAE
}

\author{
L. R. YUNGELSON and A. V. TUTUKOV \\ Institute for Astronomy, 48 Pyatnitskaya Str., 109017 Moscow, Russia
}

The present birthrate of binaries may be written as

$d^{3} \nu=0.2 M_{1}^{-2.5} q^{\alpha} d M_{1} d \log A d q \quad \mathrm{yr}^{-1}$,

where $M_{1}$ is the mass of the primary in $M_{\odot}, 0<q \leq 1$ - mass ratio of components, $A$ - semimajor axis of the orbit in $R_{\odot}$. The Eq.(1) implies that all stars are born as binaries with $10 \leq A / R_{\odot} \leq 10^{6}$, and that one binary with $M_{1} \geq 0.8 M_{\odot}$ is formed annually in the Galaxy. We study numerically evolutionary scenarii of binaries within abovementioned range of $M_{1}, A, q$. As PN formation events we consider all ejections of common envelopes by close binaries and ejections of envelopes by red giants, after which one may expect a formation of a hot $\left(T_{e} \geq 30000 \mathrm{~K}\right)$ star, surrounded by a nebula. Altogether about 20 different single and binary cores of PN can be formed. Combining the scenarii data with the Eq. (1) one can estimate the birthrates of most numerous kinds of PNN (single and with main-sequence, white dwarf, giant and relativistic companions) listed in the Table.

\begin{tabular}{|l|c|cccc|}
\multicolumn{3}{|c|}{ The birth rate of PNN in the Galaxy $\left(y r^{-1}\right)$} \\
\cline { 3 - 6 } Nucleus & Single & MS & WD & Giant & Rel \\
\hline CO, ONe dwarf & 0.17 & 0.62 & 0.08 & 0.0027 & 0.00082 \\
He dwarf & 0.0027 & 0.042 & 0.0042 & - & - \\
He star & - & 0.0014 & 0.01 & - & 0.0027 \\
\hline
\end{tabular}

The single PNN within our concept of PN formation are mainly products of the merger of components of binaries inside common envelopes.

The Figure shows the distribution over orbital periods of PNN with MS (thick line) and WD (thin line) companions, as well as positions of PNN with known orbital periods (dots). The theoretical distribution of PNN agrees with observational estimate of 10 per cent of PNN being close binaries with $P \preceq 10^{d}$. The absence of more wide, but still close, observed binary PNN is a result of observational selection, because they can be discovered mainly due to photometrical variability caused by the presence of close companion. The position of observed wide binary PNN is a result of the existence of lower angular separation limit for discovery of

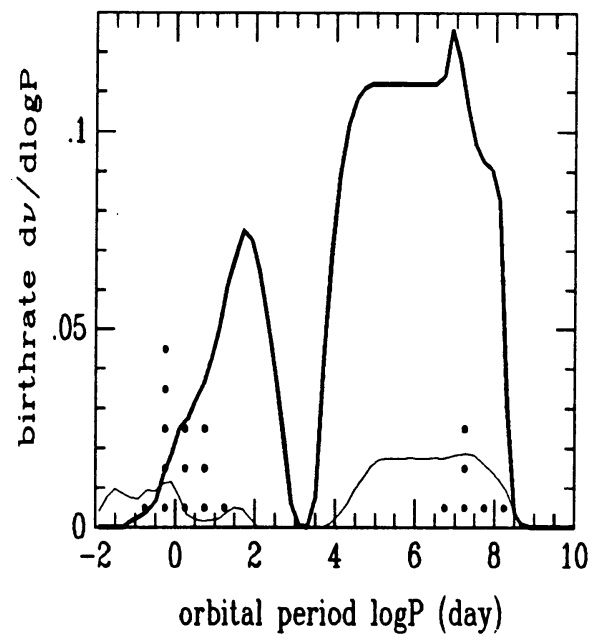
visual duplicity $\sim 1$ arcsec. 